\title{
OVERCOMING DEPOLARIZING RESONANCES WITH A SIBERIAN SNAKE
}

\author{
B. Vuaridel, A. D. Krisch, R. S. Raymond, T. Roser, and J. A. Stewart \\ Randall Lab of Physics, University of Michigan, Ann Arbor, MI 48109 USA
}

J. E. Goodwin, H-O. Meyer, M. G. Minty, P. V. Pancella,

R. E. Pollock, T. Rinckel, M. A. Ross, F. Sperisen, E. J. Stephenson and

B. VON PRZEWOSKI

Indiana University Cyclotron Facility, Bloomington, IN 47408 USA

\author{
E. D. Courant, S. Y. Lee and L. G. Ratner \\ Brookhaven National Laboratory, Upton, NY 11973 USA
}

In all circular proton accelerators there are certain energies where the spin of each proton precesses an integer number of times about the vertical axis on each turn around the ring. Any horizontal fields in the ring can then interact coherently with the spins and depolarize the beam. An arrangement of magnets, called a Siberian Snake, was proposed by Derbenev and Kondratenko ${ }^{1}$ to overcome depolarizing resonances. A Siberian Snake rotates the spin of each proton by $180^{\circ}$ forcing the effects of all depolarizing horizontal magnetic fields to cancel themselves on successive turns. In other words, the spin tune $\nu_{\text {spin }}$, which is the number of times that a proton's spin precesses in each turn, is forced by the Siberian Snake to be exactly one half, independent of energy. The condition for depolarization thus never occurs and the polarization is maintained during the acceleration.

A Siberian Snake was installed in the Indiana University Cooler Ring. We studied intrinsic and imperfection depolarizing resonances with this Snake $^{2}$. We used the ring quadrupoles to ramp the vertical betatron tune, $\nu_{y}$, through the $\mathrm{G} \gamma=-3+\nu_{\mathrm{y}}$ intrinsic depolarizing resonance. We then found significant depolarization without the Snake and no observable depolarization with the Snake turned on. This tune ramping was equivalent to accelerating through the resonance; it thus directly tested a Snake's ability to overcome a strong intrinsic depolarizing resonance. We accelerated through the $\mathrm{G} \gamma=2$ imperfection resonance and found a complete polarization reversal without the Snake. This was expected 


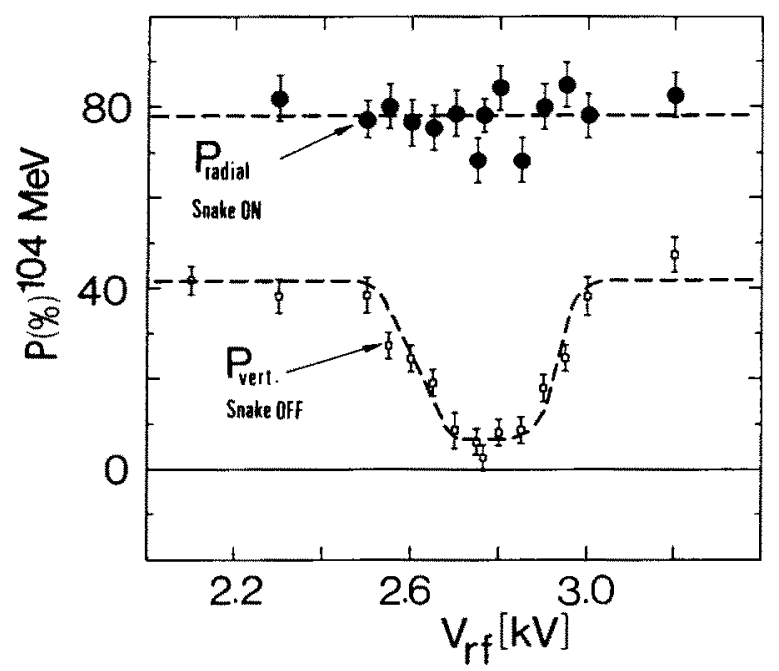

Fig. 1. The beam polarization in each stable direction at $104 \mathrm{MeV}$ is plotted against the voltage of the rf accelerating cavity in the Cooler Ring, with the Snake on and off.

in the Cooler Ring since a rapid acceleration through the resonance is not feasible. However, no spin-flip was observed with the Snake turned on.

During our investigation of the imperfection resonance we observed another type of "synchrotron" depolarizing resonance which is related to the rf accelerating voltage. We could vary this voltage and thus change the synchrotron tune, $\nu_{\text {syn }}$, which is the number of times that a proton's energy oscillates about the average cnergy in cach turn around the ring. Since a proton's orbit depends on its energy, the depolarizing magnetic fields seen by a proton vary with this oscillation frequency. As shown in Fig. 1, we observed almost. complete depolarization of the proton beam at $104 \mathrm{MeV}$ for an if accelerating voltage of about $2.8 \mathrm{kV}$. With the Snake turned on, depolarization did not occur. This appears to be an additional indication that Siberian Snakes can be used to overcome all types of depolarizing resonances. Thus it may be technically feasible to accelerate polarized beams to very high energies.

This research was supported by grants from the U. S. Department of Energy and the U.S. National Science Foundation.

References:

1. Ya. S. Derbenev and A. M. Kondratenko, Part. Accel., 8, 115 (1978).

2. A. D. Krisch et al., Phys. Rev. Lett., 63, 1137 (1989) and J. E. Goodwin et al., Phys. Rev. Lett., 64, 2779 (1990). 